\title{
ÜBER GITTERPUNKTE IN CONVEXEN KÖRPERN UND EIN DAMIT ZUSAMMENHÄNGENDES EXTREMALPROBLEM.
}

\author{
VoN \\ CARL LUDWIG SIEGEL \\ in Princeton, N. J.
}

Bekanntlich ist im n-dimensionalen Raum für jeden zum Nullpunkt symmetrischen convexen Körper $\mathfrak{\Omega}$, der keinen vom Nullpunkt verschiedenen Gitterpunkt enthält, das Volumen $V$ höchstens gleich $2^{n}$. Wie das Beispiel eines zu den Achsen parallel orientierten Würfels zeigt, lässt dieser Minkowskische Satz in bezug auf die obere Schranke für $V$ bei beliebigen Körpern keine Verschärfung zu. Betrachtet man aber aus der Menge der Körper $\mathfrak{A}$ nur einen Teil, wie etwa die $n$-dimensionalen Ellipsoide, so ergibt sich die Aufgabe, in dieser Teilmenge eine möglichst günstige obere Schranke für $V$ zu finden.

Das naturgemässe analytische Werkzeug für die Untersuchung des Zahlengitters sind die $n$-fachen Fourierschen Reihen; mit ihrer Vollständigkeitsrelation beweist man für convexe Körper, die keinen Gitterpunkt ausser dem Nullpunkt enthalten, die Beziehung

$$
2^{n}=V+V^{-1} \sum_{\mathfrak{l} \neq 0}\left|\int_{\Omega} e^{\pi i\lfloor\mathfrak{r}} d \mathfrak{x}\right|^{2},
$$

wo $\mathfrak{l}=\left(l_{1}, \ldots, l_{n}\right)$ alle vom Nullpunkt verschiedenen Gitterpunkte durchläuft und zur Abkürzung $d x_{1} \ldots d x_{n}=d \mathfrak{x}, l_{1} x_{1}+\cdots+l_{n} x_{n}=\mathfrak{l} \mathfrak{x}$ gesetzt ist. Dies setzt den Minkowskischen Satz in Evidenz.

Der zu (I) führende Ansatz lässt sich bei speciellen Typen von Körpern zur Verbesserung der oberen Schranke für $V$ verwenden. Insbesondere erhält man für Ellipsoide die obere Schranke $\left(\frac{4 \sqrt{2}}{3}\right)^{n}$ bei hinreichend grossem n. Al- 
lerdings wurde bereits auf anderem Wege durch Blichfeldt die günstigere Schranke $2^{\frac{n}{2}}\left(\frac{n}{2}+1\right)$ gefunden.

Eine Verallgemeinerung des eben erwähnten Ansatzes führt auf folgendes Variationsproblem: Man bestimme die grösste Zahl $\tau$, so dass für jede in der Form

$$
f(\mathfrak{y})=\int_{\mathfrak{r}} \varphi(\mathfrak{x}) e^{2 \pi i \mathfrak{x} \mathfrak{y}} d \mathfrak{x}
$$

darstellbare ganze Function $f(\mathfrak{y})$, die für reelles $\mathfrak{y}=\left(y_{1}, \ldots, y_{n}\right)$ reell und im Nullpunkte $=\mathrm{I}$ ist, das Erfülltsein der Ungleichung

$$
\int_{-\infty}^{+\infty} \cdots \int_{-\infty}^{+\infty} f(\mathfrak{l}) d \mathfrak{l}<\tau
$$

die Existenz einer reellen Nullstelle von $f(\mathfrak{l})$ bedingt. In anderer Formulierung: Für die Menge aller durch (2) darstellbaren Functionen $f(\mathfrak{y})$, die für reelle $\mathfrak{y}$ positiv und im Nullpunkte $=$ I sind, ermittle man die untere Schranke $\imath$ des Integrales $\int_{-\infty}^{+\infty} \ldots \int_{-\infty}^{+\infty} f(\mathfrak{y}) d \mathfrak{y}$. Es zeigt sich, dass für Ellipsoide $\tau$ den Wert $2^{n}: V$ besitzt. Dieses Resultat enthält folgenden functionentheoretischen Satz:

Es sei $f(z)$ eine ganze Function der complexen Variabeln $z$, reell für reelles $z, f(\mathrm{o})=\mathrm{I}$ und für jedes $\varrho>\mathrm{I}$ bei $z \rightarrow \infty$

$$
f(z)=O\left(e^{e|z|}\right) .
$$

Ist dann für irgend ein natürliches $n$

$$
\int_{-\infty}^{+\infty} f(x)|x|^{n-1} d x \leq 2^{2 n} \Gamma\left(\frac{n}{2}\right) \Gamma\left(\frac{n}{2}+\mathrm{I}\right)
$$

so hat $f(z)$ eine reelle Nullstelle. In dieser Aussage kann die rechte Seite von (3) durch keine grössere Zahl ersetzt werden.

\section{$\S$ I. Der Satz von Minkowski.}

Es sei $\varphi(\mathfrak{x})$ eine quadratisch integrierbare Function des Punktes $\mathfrak{x}$, die überall ausserhalb des Körpers $\mathfrak{A}$ verschwindet. Durchläuft $\mathfrak{l}$ alle Gitterpunkte, so hat die durch die Gleichung 
(4)

$$
f(\mathfrak{x})=\sum_{\mathfrak{f}} \varphi(2 \mathfrak{x}-2 \mathfrak{f})
$$

definierte quadratisch integrierbare Function $f(x)$ in jeder Coordinate $x_{h}(h=\mathrm{I}$, $\ldots, n)$ die Periode I. Bedeutet $\mathfrak{F}$ den Einheitswürfel und $\Re$ den gesamten Raum, so gilt für die Fourierschen Constanten von $f(x)$ nach (4) die Beziehung

$$
a_{\mathfrak{l}}=\int_{\mathscr{夭}} f(\mathfrak{x}) e^{-2 \pi i \mathfrak{x}} d \mathfrak{x}=\int_{\mathfrak{\Re}} \varphi(2 \mathfrak{x}) e^{-2 \pi i \mathfrak{I}} d \mathfrak{x}=2^{-n} \int_{\mathfrak{x}} \varphi(\mathfrak{x}) e^{-\pi i \mathfrak{I} \mathfrak{x}} d \mathfrak{x} .
$$

Nach dem Vollständigkeitssatz ist

$$
\int_{\widetilde{\gamma}}|f(\mathfrak{x})|^{2} d x=\sum_{\mathfrak{l}}\left|a_{\mathfrak{l}}\right|^{2}
$$

wo 〔 alle Gitterpunkte durchläuft. Für die linke Seite gilt vermöge (4)

$$
\int_{\mathfrak{S}}|f(\mathfrak{x})|^{2} d \mathfrak{x}=\sum_{\mathfrak{t}} \int_{\mathfrak{H}} \overline{\varphi(2 \mathfrak{x})} \varphi(2 \mathfrak{x}-2 \mathfrak{l}) d \mathfrak{x}=2^{-n} \sum_{\mathfrak{l}} \int_{\mathfrak{K}} \overline{\varphi(\mathfrak{x})} \varphi(\mathfrak{x}-2 \mathfrak{l}) d \mathfrak{x}
$$

und die rechte Seite lässt sich nach (5) umformen. Dies liefert

$$
\sum_{\mathfrak{t}} \int_{\mathfrak{r}} \overline{\varphi(\mathfrak{x})} \varphi(\mathfrak{x}-2 \mathfrak{f}) d \mathfrak{x}=2^{-n} \sum_{\mathfrak{l}}\left|\int_{\mathfrak{a}} \varphi(\mathfrak{x}) e^{-\pi i \mathfrak{L}} d \mathfrak{x}\right|^{2}
$$

Ist nun $\overline{\varphi(\mathfrak{x})} \varphi(\mathfrak{x}-2 \mathfrak{l}) \neq 0$, so liegen $\mathfrak{x}$ und $\mathfrak{x}-2 \mathfrak{l}$ in $\mathfrak{R}$, wegen der Symmetrie und der Convexität also auch der Gitterpunkt $\mathfrak{f}$. Enthält $\mathfrak{R}$ ausser dem Nullpunkt keinen Gitterpunkt, so reduciert sich die linke Seite von (7) auf das eine einzige Glied $\mathfrak{f}=0$, und $(7)$ geht über in

$$
\int_{\mathfrak{x}}|\varphi(\mathfrak{x})|^{2} d \mathfrak{x}=2^{-n} \sum_{l}\left|\int_{\mathfrak{R}} \varphi(\mathfrak{x}) e^{-\pi i \mathfrak{x}} d \mathfrak{x}\right|^{2}
$$

Hieraus entsteht (I), indem $\varphi(\mathfrak{x})$ innerhalb $\mathfrak{K}$ identisch gleich I gewählt wird. Es ist also $2^{n}: V$ gleich der Quadratsumme der Mittelwerte aller Grössen $e^{\text {ri } \mathfrak{I}_{\mathfrak{x}} \text { in }}$ $\Re$. Dies ist eine Verfeinerung des Minkowskischen Satzes; für die Ableitung dieses Satzes selbst genügte es, an Stelle der Vollständigkeitsrelation die Schwarzsche Ungleichung 


$$
\int_{\pi}|f(x)|^{2} d x \geq\left|a_{0}\right|^{2}
$$

zu verwenden.

\section{$\S 2 . \quad$ Ellipsoide.}

Es sei $\Re$ speciell ein Ellipsoid mit der Gleichung $P(\mathfrak{x})=\mathrm{I}$, wo $P(\mathfrak{x})$ eine positiv definite quadratische Form bedeutet. Eine einfache Rechnung zeigt dann, dass der Mittelwert von $e^{\pi i I_{x}}$ in $\mathfrak{R}$ durch $B_{\frac{n}{2}}(\pi \sqrt{Q(\mathfrak{l})})$ gegeben wird, wo $B_{\lambda}(x)$ mit der Besselschen Function $I_{\lambda}(x)$ durch die Gleichung

(9) $\quad B_{\lambda}(x)=\Gamma(\lambda+\mathrm{I})\left(\frac{x}{2}\right)^{-\lambda} I_{\lambda}(x)=\sum_{n=0}^{\infty} \frac{(-1)^{n}}{n !(\lambda+1)(\lambda+2) \ldots(\lambda+n)}\left(\frac{x}{2}\right)^{2 n}$

zusammenhängt und $Q(\mathfrak{x})$ die zu $P(\mathfrak{x})$ reciproke quadratische Form bedeutet. Also ist

$$
2^{n}: V=\mathrm{I}+\sum_{\mathfrak{l} \neq 0} B_{\frac{2}{2}}^{2}(\pi \sqrt{Q(\mathfrak{l})})
$$

Mit der in dieser Formel steckenden Verschärfung des Minkowskischen Satzes für den Fall des Ellipsoids lässt sich nicht viel anfangen, da die Aufsuchung einer positiven unteren Schranke für die Summe auf der rechten Seite Schwierigkeiten macht. $\mathrm{Zu}$ einer brauchbaren Abschätzung kommt man aber, indem man von (7) ausgeht und $\varphi(\mathfrak{x})$ genau wie oben specialisiert, unter der Voraussetzung, dass $\Re$ ausser dem Mittelpunkt noch einen weiteren Gitterpunkt enthält. Das auf der linken Seite von (7) auftretende Integral ist dann gleich dem Volumen $V_{\mathfrak{r}}$ des Durchschnitts von $\Re$ und dem durch die Translation um den Vector $2 \mathfrak{f}$ entstehenden Körper. Man erhält also die Ungleichung

$$
\sum_{\mathfrak{l}} V_{\mathfrak{l}} \geq 2^{-n} V^{2} .
$$

Für den Fall des Ellipsoids gilt speciell

$$
V_{\mathfrak{l}}=V \int_{V \frac{1}{P(\mathfrak{k})}}^{1}\left(\mathrm{I}-x^{2}\right)^{\frac{n-1}{2}} d x: \int_{0}^{1}\left(\mathrm{I}-x^{2}\right)^{\frac{n-1}{2}} d x \quad(P(\mathfrak{f}) \leq \mathrm{I}) .
$$

Enthielte das Ellipsoid $\mathfrak{R}$ mit der Gleichung $P(\mathfrak{x})=\mathrm{I}$ und dem Volumen $V$ mehr 
als $3^{n}$ Gitterpunkte, so gäbe es zwei Gitterpunkte $\mathfrak{l}$ und $\mathfrak{l}$ mit $\mathfrak{l} \equiv \mathfrak{l}(\bmod .3)$ und das Ellipsoid $P(\mathfrak{x})=\left(\frac{2}{3}\right)^{2}$ vom Volumen $\left(\frac{2}{3}\right)^{n} V$ enthielte den vom Nullpunkt verschiedenen Gitterpunkt $\frac{\mathfrak{l}-\mathfrak{l}}{3}$. Enthält nun aber das letztere Ellipsoid keinen Gitterpunkt ausser dem Nullpunkt, so ist die Anzahl der Gitterpunkte in $\mathfrak{R}$ höchstens $3^{n}$. Es bedeute $\lambda$ das Minimum der Werte $\sqrt{P(\mathfrak{f})}$ in den vom Nullpunkt verschiedenen Gitterpunkten. Dann gilt nach (II) und (12) für das Volumen des Ellipsoids $P(\mathfrak{x})=\lambda^{2}$, das ausser dem Mittelpunkt keinen Gitterpunkt im Innern besitzt, die Ungleichung

$$
V \lambda^{n} \leq(2 \lambda)^{n}\left(\mathrm{I}+\left(3^{n}-\mathrm{I}\right) \int_{\lambda}^{1}\left(1-x^{2}\right)^{\frac{n-1}{2}} d x \frac{2 \Gamma\left(\frac{n}{2}+\mathrm{I}\right)}{\pi^{\frac{1}{2}} \Gamma\left(\frac{n+\mathrm{I}}{2}\right)}\right) .
$$

Ist nun $P(\mathfrak{x})$ derart normiert, dass $\lambda=\frac{2}{3} \sqrt{2} n^{-\frac{1}{16 n}}$, also sicherlich $\frac{2}{3}<\lambda<$ I ist, so hat die rechte Seite von (13) für unendlich werdendes $n$ den asymptotischen Wert $(2 \lambda)^{n}$. Bei hinreichend grossem $n$ enthält daher ein Ellipsoid vom Volumen $\left(\frac{4 \sqrt{2}}{3}\right)^{n}$ ausser dem Mittelpunkt noch einen weiteren Gitterpunkt. Es wurde bereits in der Einleitung bemerkt, dass Blichfeldt eine noch bessere Schranke für das Volumen abgeleitet hat. Andererseits hat Minkowski mit Hilfe seiner Reductionstheorie der quadratischen Formen bewiesen, dass es $n$-dimensionale Ellipsoide vom Volumen 2 gibt, die ausser dem Mittelpunkt keinen Gitterpunkt enthalten.

\section{$\S$ 3. Das Extremalproblem.}

Es sei $\mathfrak{R}$ zunächst wieder ein beliebiger convexer Körper, der zum Nullpunkt symmetrisch liegt und sonst keinen Gitterpunkt enthält. Bedeutet $\psi(\mathfrak{x})$ eine stetige Function, die überall ausserhalb $\Re$ verschwindet, so haben die Fourierschen Constanten der periodischen Function

die Werte

$$
g(\mathfrak{x})=\sum_{\mathfrak{i}} \psi(\mathfrak{x}+\mathfrak{f})
$$




$$
b_{\mathfrak{l}}=\int_{\mathfrak{r}} \psi(\mathfrak{x}) e^{-2 \pi i \mathfrak{x}} d \mathfrak{x} .
$$

Setzt man nun voraus, dass diese Werte $b_{\mathfrak{r}}$ sämtlich $\geq 0$ sind, so wird nach dem Fejérschen Satze $g(\mathfrak{x})$ durch die Fouriersche Reihe dargestellt, und es ist speciell

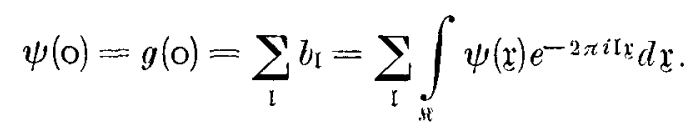

Da man hieraus (8) erhält, indem man für $\psi(\mathfrak{x})$ die Function $\int_{\mathfrak{H}} \overline{\varphi(\mathfrak{y})} \varphi(\mathfrak{y}+2 \mathfrak{d}) d \mathfrak{y}$ einsetzt, so könnte man hoffen, durch geeignete anderweitige Wahl von $\psi(\mathfrak{x})$ für den Fall des Ellipsoids eine brauchbarere Verschärfung des Minkowskischen Satzes zu finden, als sie durch (Io) gegeben wird. Um eine günstige obere Abschätzung von $V$ zu bekommen, muss man bei festem Werte von $\psi(0)$ eine günstige untere Abschätzung für die Summe der Mittelwerte der Grössen $\psi(\mathfrak{x}) e^{-2 \pi i\lceil\mathfrak{r}}$ angeben. Da diese aber wieder von den Gitterpunkten $\mathfrak{l}$ abhängen, so wird man sich mit dem Mittelwert von $\psi(\mathfrak{x})$ allein als Minorante begnügen. Da ferner in der Bedingung $b_{\mathfrak{l}} \geq 0$ auch die Gitterpunkte auftreten, so wird man sie durch die schürfere, aber bequemer zu benutzende ersetzen, dass die Function

$$
h(\mathfrak{y})=\int_{\mathfrak{a}} \psi(\mathfrak{x}) e^{-2 \pi i \mathfrak{y} y} d \mathfrak{x}
$$

im ganzen $\mathfrak{y}$-Raum $\Re$ (und nicht nur in seinen Gitterpunkten $\mathfrak{l}$ ) nicht-negativ ist. Bedeutet $\sigma$ die obere Schranke der Werte $\int_{\mathscr{H}} \psi(\mathfrak{x}) d \mathfrak{x}: \int_{\mathscr{H}} \psi(\mathrm{o}) d \mathfrak{x}$ für die Menge aller derjenigen im Ellipsoid $\mathfrak{K}$ stetigen Functionen $\psi(\mathfrak{x})$, für welche die durch (I5) definierte Function $h(\mathfrak{y})$ im ganzen $\mathfrak{y}$-Raum nicht-negativ ist, so ist nach (I4)

$$
V \leq \sigma^{-1}
$$

Es zeigt sich nun, dass $\sigma=2^{-n}$ ist; man erhält also auf diesem Wege keine Verschärfung des Minkowskischen Satzes. Da aber die zur Lösung des Extremalproblems führenden Überlegungen an sich ein gewisses Interesse besitzen und das Resultat Anwendungen auf die Theorie der ganzen Functionen gestattet, so möge der Beweis der-Gleichung $\sigma=2^{-n}$ hier mitgeteilt werden. 
I) Da jedes Ellipsoid durch eine affine Transformation in die Einheitskugel $|\mathfrak{x}|=\mathrm{I}$ verwandelt werden kann, so darf man ohne Beschränkung der Allgemeinheit voraussetzen, dass $\mathfrak{K}$ diese Kugel ist. Ferner bleibt (15) richtig, wenn darin $h(\mathfrak{y})$ und $\psi(\mathfrak{x})$ durch ihre Mittelwerte auf den zum Nullpunkt concentrischen Kugeln ersetzt werden, und dabei behält auch der Ausdruck $\int_{\Re} \psi(\mathfrak{x}) d \mathfrak{x}: \int_{\mathfrak{R}} \psi(0) d \mathfrak{x}$ seinen Wert. Folglich darf man für die Lösung des Extremalproblems voraussetzen, dass $\psi(\mathfrak{x})$. Rotationssymmetrie in bezug auf den Nullpunkt besitzt, also im wesentlichen nur von einer Variabeln abhängt. Nach (15) ist dann auch $h(\mathfrak{y})$ rotationssymmetrisch. Wegen der Voraussetzung $h(\mathfrak{n}) \geq 0$ wird umgekehrt auch die stetige Function $\psi(\mathfrak{x})$ durch das Fouriersche Integral

$$
\psi(\mathfrak{x})=\int_{\mathfrak{r}} h(\mathfrak{y}) e^{2 \pi i \mathfrak{x} \mathfrak{y}} d \mathfrak{y}
$$

dargestellt. Normiert man noch

$$
h(0)=\mathbf{I}
$$

so ist

$$
\int_{\mathfrak{x}} \psi(\mathfrak{x}) d \mathfrak{x}: \int_{\mathfrak{x}} \psi(0) d \mathfrak{x}=V^{-1}: \int_{\mathfrak{M}} h(\mathfrak{y}) d \mathfrak{y}
$$

und man hat zu beweisen, dass für die Menge aller in der Form (15) darstellbaren rotationssymmetrischen nicht-negativen Functionen $h(\mathfrak{y})$ das Integral $\int_{\Re} h(\mathfrak{y}) d \mathfrak{y}$ unter der Nebenbedingung $h(\mathrm{o})=\mathrm{I}$ die untere Schranke $\tau=V^{-1} \sigma^{-1}=$ $=2^{n}: V$ besitzt.

2) Es soll jetzt gezeigt werden, dass für eine geeignete zulïssige Function $h(\mathfrak{y})$ die untere Schranke $\tau$ wirklich angenommen wird. Es sei $\psi_{k}(\mathfrak{x})$ eine Minimalfolge, also $\psi_{k}$ rotationssymmetrisch und stetig, =0 überall ausserhalb von $\mathfrak{K}$,

$$
\int_{\mathfrak{l}: \mathfrak{i} \leq 1} \psi_{k}(\mathfrak{x}) e^{-2 \pi i \mathfrak{y} \mathfrak{y}} d \mathfrak{x}=h_{k}(\mathfrak{y})
$$

nicht-negativ in $\Re, h_{k}(\mathrm{o})=\mathrm{I}$ und

$$
\int_{\Re} h_{k}(\mathfrak{y}) d \mathfrak{y} \rightarrow \tau .
$$

40-34686. Acta mathematica. 65. Imprimé le 1 avril 1935. 
Ist $M$ die obere Schranke der linken Seite von (18), so ist nach (16)

$$
\left|\psi_{k}(\mathfrak{x})\right| \leq M
$$

Vermöge $(17)$ ist $h_{k}(\mathfrak{y})$ eine ganze Function der complexen Variabeln $\mathfrak{y}=\left(y_{1}, \ldots, y_{n}\right)$, für die wegen (19) die Ungleichung

$$
\left|h_{k}(\mathfrak{y})\right| \leq M \int_{|x| \leq 1} e^{2 \pi \mathfrak{x} I(y)} d \mathfrak{x}
$$

gilt. Die Folge der $h_{k}(\mathfrak{h})$ ist daher gleichmässig beschränkt in jedem endlichen Teile des complexen $\mathfrak{y}$-Raumes. Nach dem Häufungstellenprincip darf man dann voraussetzen, dass die Folge $h_{k}(\mathfrak{y})$ in jedem endlichen Teile des complexen $\mathfrak{y}$ Raumes gleichmässig gegen eine Grenzfunction $h(\mathfrak{h})$ convergiert. Es ist auch $h(\mathfrak{y})$ nicht-negativ für reelle $\mathfrak{y}$, rotationssymmetrisch in bezug auf $\mathfrak{y}=0$ und $h(0)=\mathbf{I}$. Bedeutet ferner $\mathfrak{R}^{\prime}$ irgend einen endlichen Teil des reellen $\mathfrak{y}$-Raumes, so ist nach ( 18 )

$$
\int_{\mathfrak{\mathfrak { N } ^ { \prime }}} h_{k}(\mathfrak{y}) d \mathfrak{y} \leq \int_{\mathfrak{M}} h_{k}(\mathfrak{y}) d \mathfrak{y} \rightarrow \boldsymbol{\tau}
$$

und andererseits

$$
\int_{\mathfrak{Y}^{\prime}} h_{k}(\mathfrak{y}) d \mathfrak{y} \rightarrow \int_{\mathfrak{x}^{\prime}} h(\mathfrak{y}) d \mathfrak{y},
$$

also

$$
\int_{\mathfrak{M}} h(\mathfrak{y}) d \mathfrak{y} \leq \tau
$$

Um von der Function $h(\mathfrak{y})$ nachzuweisen, dass sie in der Form (1 5 ) dargestellt werden kann, bilde man die durch (I6) definierte Function $\psi(\mathfrak{x})$ der reellen Variabeln $x$. Es soll gezeigt werden, dass sie überall ausserhalb der Einheitskugel verschwindet.

Es sei $\mathfrak{x}$ ein Punkt ausserhalb von $\mathfrak{K}$. Man wähle $n$ positive Zahlen $\xi_{1}, \ldots, \xi_{n}$ so klein, dass auch noch jeder Punkt $t$ des Rechtflachs $x_{k} \leq t_{k} \leq x_{k}+\xi_{k}(k=\mathrm{I}, \ldots, n)$ ausserhalb von $\mathfrak{R}$ liegt. Da alle $\psi_{k}(\mathfrak{x})$ im ganzen Äussern von $\mathfrak{R}$ verschwinden, so ist nach (I6)

$$
\int_{\mathfrak{r}} h_{k}(\mathfrak{y}) e^{2 \pi i \mathfrak{y}} d \mathfrak{y}=\mathrm{o}
$$


and hieraus folgt durch Integration über das Rechtflach

$$
\int_{\Re} h_{k}(\mathfrak{y}) e^{2 \pi i \mathfrak{x} \mathfrak{y}} \prod_{r=1}^{n} \frac{e^{2 \pi i \check{s}_{r} y_{r}}-\mathrm{I}}{2 \pi i y_{r}} d \mathfrak{y}=0 .
$$

Da nun in jedem endlichen Teil von $\mathfrak{R}$ die Folge $h_{k}(\mathfrak{y})$ gleichmässig gegen $h(\mathfrak{y})$ strebt, so liefert (2I) in Verbindung mit (18), dass auch

$$
\int_{\Re} h(\mathfrak{y}) e^{2 \pi i \mathfrak{x} y} \prod_{r=1}^{n} \frac{e^{2 \pi i \xi_{r} y_{r}}-\mathrm{I}}{2 \pi i y_{r}} d \mathfrak{y}=\mathrm{O}
$$

ist, und hieraus erhält man durch Differentiation nach $\xi_{1}, \ldots, \xi_{n}$ die Gleichung

$$
\int_{\mathfrak{R}} h(\mathfrak{y}) e^{2 \pi i \mathfrak{x}^{y}} d \mathfrak{y}=0 \quad(|\mathfrak{x}|>1)
$$

also das behauptete Verschwinden der durch (I6) definierten Function $\psi(\mathfrak{x})$ ausserhalb von $\mathfrak{R}$.

Als analytische Function hat nun $h(\mathfrak{y})$ Ableitungen aller Ordnung; nach dem Fourierschen Satz gilt also auch die Darstellung (I 5) für $h(\mathfrak{h})$. Daher ist $h(\mathfrak{y})$ eine zulässige Function, und man hat nach (20) in der Tat die Gleichung

$$
\int_{\Re} h(\mathfrak{y}) d \mathfrak{y}=\boldsymbol{\tau}
$$

3) Die gefundene Extremalfunction $h(\mathfrak{y})$ ist ganz und rotationssymmetrisch, also eine gerade ganze Function von $\sqrt{y_{1}^{2}+\cdots+y_{n}^{2}}=z$ allein. Setzt man

$$
h(\mathfrak{y})=f(z),
$$

so ist $f(0)=\mathrm{I}$ und $f(z) \geq 0$ für reelle $z$. Alle etwaigen reellen Nullstellen von $f(z)$ haben demnach gerade Ordnung. Es wird bewiesen werden, dass $f(z)$ keine nicht-reelle Nullstelle besitzt. Daraus folgt dann, dass $h(\mathfrak{y})$ das Quadrat einer ganzen Function ist.

Wäre $i \zeta$, also auch $-i \zeta$, eine rein imaginäre Nullstelle von $f(z)$, so betrachte man die ganze Function

$$
f_{1}(z)=f(z):\left(1+\frac{z^{2}}{\zeta^{2}}\right)
$$


Für diese wärø $f_{1}(0)=\mathrm{I}, f_{1}(z) \geq 0$ auf der reellen Achse,

$$
\int_{\Re} f_{1}(|\mathfrak{y}|) d \mathfrak{y}<\int_{\Re} f(|\mathfrak{y}|) d \mathfrak{y}=\boldsymbol{\tau},
$$

und dies wäre ein Widerspruch, wenn man noch zeigen kann, dass auch $f_{1}(z)$ in der Form (I 5) darstellbar wäre. $\mathrm{Zu}$ diesem Zwecke hat man wieder nur nachzuweisen, dass die Function

$$
\int_{\mathfrak{H}} \frac{h(\mathfrak{y})}{\zeta^{2}+|\mathfrak{y}|^{2}} e^{2 \pi i \mathfrak{x} \mathfrak{y}} d \mathfrak{y}=\varphi_{1}(\mathfrak{x})
$$

überall ausserhalb $\mathfrak{R}$ verschwindet. Integriert man (23) zweimal über jede Coordinate, so entsteht die Gleichung

$$
\begin{aligned}
& \int_{\Re} \frac{h(\mathfrak{y})}{\zeta^{2}+|\mathfrak{y}|^{2}} e^{2 \pi i \xi y} \prod_{r=1}^{n}\left(\frac{\sin \pi \xi_{r} y_{r}}{\pi y_{r}}\right)^{2} d \mathfrak{y}= \\
& =\int_{-\xi_{1}}^{+\xi_{1}} \ldots \int_{-s_{n}}^{+\xi_{n}} \varphi_{1}(\mathrm{x}+\mathrm{t}) \prod_{r=1}^{n}\left(\xi_{r}-\left|t_{r}\right|\right) d t_{1} \ldots d t_{n} .
\end{aligned}
$$

An Stelle des Integrals $K$ auf der linken Seite werde das Integral $K(Y)$ mit den Grenzen $y_{1}= \pm n Y, y_{2}= \pm Y, y_{3}= \pm Y, \ldots, y_{n}= \pm Y$ betrachtet, das für $Y \rightarrow \infty$ gegen $K$ convergiert. Nach dem Satz von Cauchy darf man die Integrationsstrecke $-n Y \leq y_{1} \leq+n Y$ durch den Halbkreis $y_{1}=n Y e^{i \vartheta} \quad(\pi \geq \vartheta \geq 0)$ ersetzen. In dem so abgeänderten Integrationsgebiet ist nach ( I 5), (I6) und (22)

$$
|h(\mathfrak{y})| \leq \tau \int_{|\mathfrak{x}| \leq 1} e^{2 \pi \mathfrak{g} I(\mathfrak{y})} d \mathfrak{x} \leq \tau V e^{2 \pi I\left(y_{1}\right)} .
$$

Wegen der Rotationssymmetrie genügt es, das Verschwinden von $\varphi_{1}(\mathfrak{x})$ für $x_{1}>\mathrm{I}$, $x_{2}=0, x_{3}=0, \ldots, x_{n}=0$ zu beweisen. Es ist aber nach (25) für $Y \rightarrow \infty$

$$
K(Y)=O\left(Y^{-3} \int_{0}^{\pi} e^{-2 \pi\left(x_{1}-\check{\zeta}_{1}-1\right) I\left(y_{1}\right)} d \vartheta\right)
$$

und die rechte Seite strebt gegen $o$, wenn die positive Zahl $\xi_{1} \leq x_{1}-$ I gewählt wird. Unter dieser Bedingung verschwindet also die rechte Seite von (24). Differen- 
tiiert man sie zweimal nach jeder der Variabeln $\xi_{1}, \ldots, \xi_{n}$ und lässt dann diese gegen o convergieren, so folgt die Behauptung.

Eine rein imaginäre Nullstelle von $f(z)$ existiert daher nicht. Besässe andererseits $f(z)$ eine Nullstelle $\zeta=\xi+i \eta$ mit $\xi \eta \neq 0$, so wäre, da. $f(z)$ gerade und auf der reellen Achse reell ist, auch $\pm \xi \pm i \eta$ eine Nullstelle. Die ganze Function

$$
f_{2}(z)=\frac{\left(\mathrm{I}-\frac{z^{2}}{|\zeta|^{2}}\right)^{2} f(z)}{\left(\mathrm{I}-\frac{z^{2}}{\zeta^{2}}\right)\left(\mathrm{I}-\frac{z^{2}}{\bar{\zeta}^{2}}\right)}
$$

wo $\bar{\zeta}$ die zu $\zeta$ conjugiert complexe Zahl $\xi-i \eta$ bedeutet, ist dann I für $z=0$ und nicht-negativ für reelle $z$. Die Darstellbarkeit von $f_{2}(z)$ in der Form (I5) folgt auf dieselbe Weise wie oben die von $f_{1}(z)$. Da nun aber für reelles $z \neq 0$ die Ungleichung

$$
\begin{aligned}
\left(1-\frac{z^{2}}{|\zeta|^{2}}\right)^{2}=1-z^{2}\left(\frac{I}{|\zeta|^{2}}+\frac{1}{|\zeta|^{2}}\right)+\frac{z^{4}}{|\zeta|^{4}}<I-z^{2}\left(\frac{1}{\zeta^{2}}+\frac{I}{\bar{\zeta}^{2}}\right)+ & \\
& +\frac{z^{4}}{\zeta^{2} \bar{\zeta}^{2}}=\left(1-\frac{z^{2}}{\zeta^{2}}\right)\left(1-\frac{z^{2}}{\bar{\zeta}^{2}}\right)
\end{aligned}
$$

gilt, so folgte

$$
\int_{\mathfrak{R}} f_{\mathfrak{2}}(|\mathfrak{y}|) d \mathfrak{y}<\int_{\mathfrak{n}} f(|\mathfrak{y}|) d \mathfrak{y}=\boldsymbol{\tau},
$$

also ein Widerspruch gegen die Extremaleigenschaft von $h(\mathfrak{y})$.

Daher ist wirklich, wie behauptet wurde, $h(\mathfrak{h})$ das Quadrat einer ganzen Function $p(\mathfrak{b})$. Wegen $h(\mathrm{o})=\mathrm{I}$ ist $p(0)= \pm \mathrm{I}$ und die Normierung $p(0)=\mathrm{I}$ zulässig.

4) Aus der Convergenz von

$$
\int_{\mathfrak{H}} p^{2}(\mathfrak{y}) d \mathfrak{y}=\boldsymbol{x}
$$

ergibt sich nach dem von Plancherel in allgemeinster Form bewiesenen Fourierschen Satz, dass das Integral

$$
\int_{\mathfrak{n}} p(\mathfrak{n}) e^{2 \pi i \mathfrak{n}} d \mathfrak{n}
$$


für fast alle $\mathfrak{x}$ convergiert und eine quadratisch integrierbare Function $\chi(\mathfrak{x})$ darstellt, die der Gleichung

$$
\int_{\mathfrak{\Re}}|\chi(\mathfrak{x})|^{2} d \mathfrak{x}=\int_{\mathfrak{H}}|p(\mathfrak{y})|^{2} d \mathfrak{y}
$$

und für fast alle $\mathfrak{y}$ der Gleichung

$$
\int_{\mathfrak{H}} \chi(\mathfrak{x}) e^{-2 \pi i \mathfrak{x} y} d \mathfrak{x}=p(\mathfrak{y})
$$

genügt. Es soll gezeigt werden, dass $\chi(x)$ fast überall im Äussern der Kugel $|x| \leq \frac{1}{2}$ verschwindet. $Z u$ diesem Zwecke sind einige der zum Plancherelschen Satz führenden Ủberlegungen im vorliegenden Fall anzuwenden.

Es sei $o<r<R$ und

$$
\int_{r \leq|\mathfrak{y}| \leq R} p(\mathfrak{y}) e^{2 \pi i \mathfrak{y} y} d \mathfrak{y}=\varphi(\mathfrak{x}, r, R)
$$

Da $p(\mathfrak{y})$ analytisch ist, so gilt für reelle $\mathfrak{y}$ umgekehrt

$$
\int_{\mathfrak{l}} \varphi(\mathfrak{s}, r, R) e^{-2 \pi i \mathfrak{y} y} d \mathfrak{x}= \begin{cases}p(\mathfrak{y}) & (r<|\mathfrak{y}|<R) \\ 0 & (|\mathfrak{y}|<r \text { oder }|\mathfrak{y}|>R)\end{cases}
$$

und ferner

$$
\int_{\mathfrak{H}}|\varphi(\mathfrak{x}, r, R)|^{2} d \mathfrak{x}=\int_{r \leq|\mathfrak{y}| \leq R}|p(\mathfrak{y})|^{2} d \mathfrak{y} .
$$

Da das letzte Integral für $r \rightarrow \infty$ den Grenzwert 0 hat, so folgt in üblicher Weise die Existenz einer monoton gegen $\infty$ wachsenden Zahlfolge $r_{k}$ von der Art, dass die Functionenfolge

$$
\int_{|\mathfrak{y}| \leq r_{k}} p(\mathfrak{y}) e^{2 \pi i \mathfrak{x} \mathfrak{d}} d \mathfrak{y}=\chi_{k}(\mathfrak{x}) \quad(k=\mathrm{I}, 2, \ldots)
$$

fast überall im $\mathfrak{x}$-Raum gegen eine Function $\chi(\mathfrak{x})$ convergiert, und zwar ist dies gerade die oben im Wortlaut des Plancherelschen Satzes auftretende Function. Aus (29) bilde man die zu (24) analoge Gleichung. 


$$
\begin{aligned}
\int_{|\mathfrak{y}| \leq r_{k}} p(\mathfrak{y}) e^{2 \pi i \mathfrak{r} \mathfrak{y}} \prod_{s=1}^{n}\left(\frac{\sin \pi \xi_{s} y_{s}}{\hbar y_{s}}\right)^{2} d \mathfrak{y}= & \\
= & \int_{-\xi_{1}}^{+\xi_{1}} \cdots \int_{-\xi_{n}}^{+\xi_{n}} \chi_{k}(\mathfrak{x}+\mathfrak{t}) \prod_{s=1}^{n}\left(\xi_{s}-\left|t_{s}\right|\right) d t_{1} \ldots d t_{n}
\end{aligned}
$$

Setzt man zur Abkürzung $r_{k}^{2}-\left(y_{2}^{2}+\cdots+y_{n}^{2}\right)=Y^{2}$, so hat man bei festen $y_{2}, \ldots, y_{n}$ über $y_{1}$ von $-Y$ bis $+Y$ zu integrieren. Nach dem Satze von Cauchy kann man statt dessen über den Halbkreis $y_{1}=Y e^{i \vartheta}(\pi \geq \vartheta \geq 0)$ integrieren. Da nun nach (25) im Integrationsgebiet die Ungleichung

$$
|p(\mathfrak{y})| \leq \sqrt{\tau} \bar{V} e^{\pi I\left(y_{1}\right)}
$$

gilt, so kann man für wachsendes $k$ das Integral auf der linken Seite von (30) dureh

$$
O\left(\int \cdots \int_{\substack{2 \\ y_{2}^{2}+\cdots+y_{n}^{2} \leq r_{k}^{2}}}^{n}\left(1+y_{s}^{2}\right)^{-1} \underset{I}{Y}+Y^{2}\left(\int_{0}^{\pi} e^{-2 \pi\left(x_{1}-\xi_{1}-\frac{1}{2}\right) I\left(y_{1}\right)} d \vartheta\right) d y_{2} \ldots d y_{n}\right)
$$

majorisieren. Wegen der Rotationssymmetrie von $p(\mathfrak{y}), \chi(x), \chi_{k}(\mathfrak{x})$ genügt es, das behauptete Verschwinden von $\chi(x)$ für $|x|>\frac{1}{2}$ unter der speciellen Annahme $x_{1}>\frac{\mathrm{I}}{2}, x_{2}=0, x_{3}=0, \ldots, x_{n}=0$ nachzuweisen. Wählt man nun die positive Zahl $\xi_{1} \leq x_{1}-\frac{I}{2}$, so strebt der Ausdruck in (3I) mit wachsendem $k$ gegen $\circ$, und dasselbe gilt also für die rechte Seite von (30). Da nun andererseits $\chi_{k}(\mathfrak{x})$ im Mittel gegen $\chi(x)$ convergiert, so folgt mit Hilfe der Schwarzschen Ungleichung die Beziehung

$$
\int_{-\xi_{1}}^{+\xi_{1}} \cdots \int_{-\xi_{n}}^{+\xi_{n}} \chi(\mathrm{x}+\mathrm{t}) \prod_{s=1}^{n}\left(\xi_{s}-\left|t_{s}\right|\right) d t_{1} \ldots d t_{n}=0
$$

und hieraus durch zweimalige Differentiation nach jeder Variabeln $\xi_{1}, \ldots, \xi_{n}$, dass $\chi(x)$ fast überall ausserhalb der Kugel $|x| \leq \frac{I}{2}$ verschwindet. 
Setzt man noch $\chi\left(\frac{\mathfrak{x}}{2}\right)=\varphi(\mathfrak{x})$, so ist also nach $(26)$ und $(27)$

$$
\int_{\mathfrak{x}}|\varphi(\mathfrak{x})|^{2} d \mathfrak{x}=2^{n} \tau
$$

und nach (28) fast überall

$$
p(\mathfrak{y})=2^{-n} \int_{\mathfrak{r}} \varphi(\mathfrak{x}) e^{-r i \mathfrak{x} \mathfrak{y}} d \mathfrak{x} .
$$

Da $p(\mathfrak{y})$ analytisch ist, so gilt die letzte Gleichung sogar überall, und es gilt speciell wegen $p(0)=\mathrm{I}$ die Formel

$$
\int_{\Re} \varphi(\mathfrak{x}) d \mathfrak{x}=2^{n}
$$

5) Nach der Schwarzschen Ungleichung ist

$$
\left|\int_{\mathfrak{x}} \varphi(\mathfrak{x}) d \mathfrak{x}\right|^{2} \leq V \int_{\mathfrak{S E}}|\varphi(\mathfrak{x})|^{2} d \mathfrak{x}
$$

wo das Gleichbeitszeichen nur dann steht, wenn $\varphi(\mathfrak{E})$ fast überall in $\mathfrak{R}$ constant ist. Aus (32) und (34) folgt also wegen der Bedeutung von $\tau$ das Resultat $\tau=2^{n}: V$, wie früher behauptet worden war. Ferner ist $\varphi(\mathfrak{x})$ fast überall in $\mathfrak{N}$ constant, und zwar ist dieser constante Wert wegen (34) gleich $2^{n}: V$. Nach (33) ist daher

$$
p(\mathfrak{y})=V^{-1} \int_{\mathfrak{x}} e^{-\pi i \mathfrak{y} y} d \mathfrak{x}
$$

oder, wie eine einfache Umformung zeigt, nach (9)

$$
\begin{aligned}
& p(\mathfrak{y})=\underset{2}{B_{n}(\pi z)} \quad\left(z^{2}=y_{1}^{2}+\cdots+y_{n}^{2}\right), \\
& h(\mathfrak{y})=\underset{2}{B_{n}^{2}(\pi z) .}
\end{aligned}
$$

Damit ist das Variationsproblem gelöst. Es hat sich genau eine rotationssymmetrische Extremalfunction ergeben. Wegen der unter 3) abgeleiteten Eigenschaften ist zugleich ohne Benutzung der Differentialgleichung oder expliciter 
Darstellungen die Realität sämtlicher Nullstellen der Besselschen Functionen $I_{n}(x)$ bewiesen. Dieser Realitätsbeweis scheint aber nicht auf allgemeinere Clas$\overline{2}$ sen von Functionen übertragbar zu sein.

\section{§ 4. Anwendungen auf die Theorie der ganzen Functionen.}

Die im vorigen Paragraphen betrachteten Functionen $h(\mathfrak{y})$ waren rotationssymmetrisch, in der Form

$$
h(\mathfrak{y})=\int_{|\mathfrak{x}| \leq 1} \psi(\mathfrak{x}) e^{-2 \pi i \mathfrak{x} \mathfrak{y}} d \mathfrak{x}
$$

darstellbar, für reelle $\mathfrak{y}$ nicht-negativ und lieferten ein convergentes Integral $\int_{\Re} h(\mathfrak{y}) d \mathfrak{y}$. Setzt man wieder $y_{1}^{2}+\cdots+y_{n}^{2}=z^{2}$ und $h(\mathfrak{y})=f(z)$, so ist $f(z)$ eine gerade ganze Function, auf der reellen Achse $f(z) \geq 0$ und das Integral $\int_{0}^{\infty} f(x) x^{n-1} d x=$ $=\frac{\mathrm{I}}{n V} \int_{\Re} h(\mathfrak{y}) d \mathfrak{y}$ convergent. Aus (35) folgt

$$
f(z)=O\left(e^{2 \pi|\Sigma|}\right) \quad(z \rightarrow \infty)
$$

Es soll nun gezeigt werden, dass (36) auch hinreichend ist, damit eine gerade ganze Function $f(z)$, für welche das reell erstreckte Integral $\int_{0}^{\infty}|f(x)| x^{n-1} d x$ convergiert, in der Form (35) dargestellt werden kann.

Auf grund des Fourierschen Satzes genügt es zu beweisen, dass die durch die Gleichung

$$
\int_{\Re} f(z) e^{2 \pi i \mathfrak{x} \mathfrak{y}} d \mathfrak{y}=\psi(\mathfrak{x}) \quad\left(z^{2}=y_{1}^{2}+\cdots+y_{n}^{2}\right)
$$

definierte Function $\psi(\mathfrak{x})$ für $|\mathfrak{x}|>\mathrm{I}$ verschwindet. Dies folgt wie das Entsprechende für $\varphi_{1}(\mathfrak{x})$ in $\S 3$ unter 3 ), falls nur die Abschätzung:

$$
f(z)=O\left(e^{2 \pi \sqrt{\left(I_{y_{1}}\right)^{2}+\cdots+\left(I y_{n}\right)^{2}}}\right) \quad(z \rightarrow \infty)
$$

41-34686. Acta mathematica. 65. Imprimó lo 1 avri] 1935. 
bewiesen werden kann. Wegen der für jedes complexe $\alpha$ gültigen Gleichung $|\alpha|^{2}=R\left(\alpha^{2}\right)+2(I \alpha)^{2}$ und der Ungleichung $|z|^{2} \leq\left|y_{1}\right|^{2}+\cdots+\left|y_{n}\right|^{2}$ ist (37) bewiesen, sowie man die Gültigkeit der schärferen Abschätzung

$$
f(z)=O\left(e^{2 \pi|r(z)|}\right) \quad(z \rightarrow \infty)
$$

gezeigt hat. Es sei nun

$$
\int_{0}^{\tilde{s}} f(\zeta) \zeta^{n-1} d \zeta=z^{n-1} g(z)
$$

Dann ist nach $(36)$ auch die ganze Function $g(z)=O\left(e^{2 \pi \mid z I}\right)$; ferner ist $g(z)$ wegen der Convergenz von $\int_{0}^{\infty}|f(x)| x^{n-1} d x$ auf der ganzen reellen Achse beschränkt. Nach einer bekannten zuerst von Lindelöf und Phragmén benutzten Schlussweise folgt daraus $g(z)=O\left(e^{2 \tau|I(z)|}\right)$. Da aber $f(z) z^{n-1}=D\left(z^{n-1} g(z)\right)$ ist, so liefert der Cauchysche Satz die Behauptung (38).

Die Voraussetzung (36) kann noch durch die schwächere ersetzt werden, dass für jedes $\varrho>2 \pi$ die Beziehung

$$
f(z)=O\left(e^{0|z|}\right) \quad(z \rightarrow \infty)
$$

gilt. Nach dem oben Bewiesenen verschwindet dann nämlich die Function

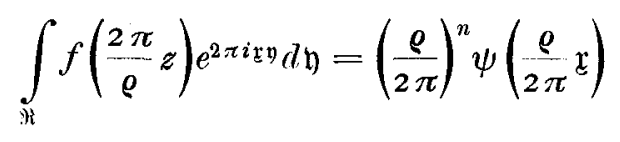

für $|\mathfrak{x}|>$ I, also auch $\psi(\mathfrak{x})$ selbst.

Beachtet man jetzt noch, dass die Function $f(z)+f(-z)$ auch für nichtgerades $f(z)$ gerade ist, so lässt sich das Resultat von $\$ 3$ folgendermassen formulieren:

Es sei $f(z)$ ganz, reell für reelles $z, f(0)=\mathrm{I}$ und für jedes $\varrho>\mathrm{I}$

Ist dann

$$
f(z)=O\left(e^{e|z|}\right) \quad(z \rightarrow \infty) .
$$

$$
\int_{-\infty}^{+\infty} f(x)|x|^{n-1} d x \leq 2^{2 n} \Gamma\left(\frac{n}{2}\right) \Gamma\left(\frac{n}{2}+\mathrm{I}\right)
$$


so hat $f(z)$ eine reelle Nullstelle, und zwar von ungerader Ordnung, wenn nicht $f(z)$ die specielle Function $B_{\frac{n}{2}}^{2}\left(\frac{z}{2}\right)$ ist. In dieser A ussage kann die rechte Seite von (39) durch keine grössere Zahl ersetzt werden.

Für die Fälle $n=\mathrm{I}, 2,3$ erhält man speciell: Es sei $f(z)$ ganz, nicht-negativ für reelles $z, f(0)=\mathrm{I}$ und $f(z)=O\left(e^{\varrho|z|}\right)$ für jedes $\varrho>\mathrm{I}$. Dann ist

$$
\int_{-\infty}^{+\infty} f(x) d x \geq 2 \pi, \quad \int_{-\infty}^{+\infty} f(x)|x| d x \geq \mathrm{1} \sigma, \quad \int_{-\infty}^{+\infty} f(x) x^{2} d x \geq 24 \pi
$$

und hierin steht das Gleichheitszeichen nur für die speciellen Functionen

$$
\begin{gathered}
f(z)=\left(\sin \frac{z}{2}\right)^{2}:\left(\frac{z}{2}\right)^{2}, \quad f(z)=\left(2 I_{1}\left(\frac{z}{2}\right)\right)^{2}:\left(\frac{z}{2}\right)^{2} \\
f(z)=\left(3 \sin \frac{z}{2}-3 \frac{z}{2} \cos \frac{z}{2}\right)^{2}:\left(\frac{z}{2}\right)^{6}
\end{gathered}
$$

Endlich ist in den Überlegungen dieses Paragraphen noch folgender Satz enthalten: Ist $f(z)$ ganz, $=O\left(e^{\varepsilon|z|}\right)$ für jedes positive $\varepsilon$ und das Integral $\int_{-\infty}^{+\infty}|f(x)| d x$ convergent, so verschwindet $f(z)$ identisch. 